Documentation et bibliothèques

\title{
Rénover ou construire pour mieux les accueillir : les retombées de l'aménagement des bibliothèques sur les usagers des bibliothèques publiques au Québec
}

\section{Marie-Ève Berthiaume}

Volume 66, numéro 1, janvier-mars 2020

Les publics : les connaître et les accueillir

URI : https://id.erudit.org/iderudit/1068826ar

DOI : https://doi.org/10.7202/1068826ar

\section{Aller au sommaire du numéro}

\section{Éditeur(s)}

Association pour l'avancement des sciences et des techniques de la documentation (ASTED)

\section{ISSN}

0315-2340 (imprimé)

2291-8949 (numérique)

Découvrir la revue

\section{Citer cet article}

Berthiaume, M.-È. (2020). Rénover ou construire pour mieux les accueillir : les retombées de l'aménagement des bibliothèques sur les usagers des bibliothèques publiques au Québec. Documentation et bibliothèques, 66(1), 30-38. https://doi.org/10.7202/1068826ar

\section{Résumé de l'article}

Les données sur les retombées des bibliothèques rénovées en sol québécois sont rarement mises à la disposition du public et des autres bibliothèques. Ainsi, l'impact des choix architecturaux et ergonomiques reste méconnu. Comment donc mesurer la valeur ajoutée pour les usagers, indépendamment de la valeur architecturale du bâtiment?

L'article présente une revue de la littérature des bonnes pratiques en aménagement des bibliothèques, suivie d'une analyse de douze projets de réaménagements de bibliothèques publiques québécoises entre 2007 et 2017, selon une méthodologie qualitative axée sur une approche interprétative des ressources documentaires et des statistiques.

Les résultats de l'analyse démontrent qu'il est possible de mettre la valeur architecturale de la bibliothèque publique au service des usagers. La valeur ajoutée pour la population se manifeste principalement par l'augmentation du nombre de visites, d'emprunts en bibliothèque, d'usagers inscrits, d'activités et de participants à celles-ci.

Parmi les recommandations, citons la collecte, la comparaison et la diffusion de statistiques avant, pendant et après les travaux comme partie intégrante de la conception architecturale et du programme fonctionnel et technique ; la segmentation par succursale des statistiques et la cohérence entre les éléments compilés lors de l'Enquête annuelle sur les bibliothèques publiques du Québec.
Tous droits réservés (C) Association pour l'avancement des sciences et des techniques de la documentation (ASTED), 2020
Ce document est protégé par la loi sur le droit d'auteur. L’utilisation des services d'Érudit (y compris la reproduction) est assujettie à sa politique d'utilisation que vous pouvez consulter en ligne.

https://apropos.erudit.org/fr/usagers/politique-dutilisation/ 


\title{
RÉNOVER OU CONSTRUIRE POUR
}

MIEUX LES ACCUEILLIR: LES RETOMBÉES

DE L'AMÉNAGEMENT DES BIBLIOTHĖQUES

SUR LES USAGERS DES BIBLIOTHĖQUES

PUBLIQUES AU QUÉBEC

\author{
Marie-Ève BERTHIAUME \\ Bibliothécaire, services au public \\ Bibliothèque publique de Drummondville \\ marie-eve.berthiaume@ville.drummondville.qc.ca
}

Les données sur les retombées des bibliothèques rénovées en sol québécois sont rarement mises à la disposition du public et des autres bibliothèques. Ainsi, l'impact des choix architecturaux et ergonomiques reste méconnu. Comment donc mesurer la valeur ajoutée pour les usagers, indépendamment de la valeur architecturale du bâtiment?

L'article présente une revue de la littérature des bonnes pratiques en aménagement des bibliothèques, suivie d'une analyse de douze projets de réaménagements de bibliothèques publiques québécoises entre 2007 et 2017, selon une méthodologie qualitative axée sur une approche interprétative des ressources documentaires et des statistiques.

Les résultats de l'analyse démontrent qu'il est possible de mettre la valeur architecturale de la bibliothèque publique au service des usagers. La valeur ajoutée pour la population se manifeste principalement par l'augmentation du nombre de visites, d'emprunts en bibliothèque, d'usagers inscrits, d'activités et de participants à celles-ci.

Parmi les recommandations, citons la collecte, la comparaison et la diffusion de statistiques avant, pendant et après les travaux comme partie intégrante de la conception architecturale et du programme fonctionnel et technique; la segmentation par succursale des statistiques et la cohérence entre les éléments compilés lors de l'Enquête annuelle sur les bibliothèques publiques du Québec.
The data on the benefits of renovated Quebec libraries are rarely available to the public and other libraries. Thus, the impact of architectural and ergonomic choices remains unrecognized. How can we measure the added value for patrons, independently to the architectural value of the building?

This article presents a literature review on best practices for interior planning and design of libraries, followed by an analysis of twelve remodeling library projects in Quebec that occurred between 2007 and 2017, following a qualitative methodology based on an interpretative approach of the documentary resources and statistics.

The results of the analysis demonstrate that is it possible to add the architectural value of the public library back to the patrons. The added value for the population is mainly manifested by an increased number of visits, in-library loans, registered users, activities, and participants to these same activities.

Among the recommendations, we mention the collection, comparison and dissemination of the statistics before, during and after the renovations as an integral part of the architectural conception and of the functional and technical program; the segmentation of the statistics and the coherence among the compiled elements by branch during the annual Inquiry on Quebec public libraries. 


\section{Introduction}

Depuis l'ouverture de la Grande Bibliothèque le 30 avril 2005, près de 45 bibliothèques publiques ont été rénovées ou construites au Québec. De ce nombre, 28 bibliothèques québécoises ont subi des rénovations modifiant l'accès aux lieux physiques, aux installations, à la collection et aux activités. Il y a fort à parier que les bibliothèques aient noté des changements sur les plans de la fréquentation, de la participation aux activités et de l'emprunt de documents à la suite de ces réaménagements.

Or, à ce jour, l'impact des rénovations sur les statistiques des bibliothèques publiques reste peu documenté au Québec, comme ailleurs au Canada et aux États-Unis. Tandis que les ouvrages traitant des bonnes pratiques architecturales pour les bibliothèques abondent, les informations et les recommandations qu'ils contiennent restent applicables dans des contextes politiques et géographiques précis, ce qui complexifie leur utilisation intégrale au Québec. Cela dit, quel que soit son lieu, le point de départ de tout projet architectural en bibliothèque demeure ancré dans le concept initial, autrement dit dans la vision que la direction de la bibliothèque transmet aux architectes et la manière dont ceux-ci l'interpréteront spatialement. Ainsi, les fondements de l'architecture des bibliothèques s'appuient sur des principes généraux mis au diapason de l'ère et du lieu où les bibliothèques sont bâties ou rénovées.

La collecte de données sur les retombées des pratiques architecturales des bibliothèques rénovées (ou construites) en sol québécois serait donc des plus pertinentes. Leur quasiinexistence a de quoi surprendre et suscite en outre des questions qui ont servi de point de départ à cette analyse. Comment pouvons-nous mettre la valeur architecturale de la bibliothèque publique au service des usagers? Au-delà de l'attrait esthétique d'un projet de réaménagement, quelle est la valeur ajoutée pour les usagers et de quelle manière pouvons-nous la mesurer? Une augmentation ou une diminution de la fréquentation de la bibliothèque, des nombres de prêts de documents et de participants aux activités a-t-elle été observée? Si oui, dans quelle mesure?

C'est dans cette optique que les objectifs de la recherche se dessinent. D'emblée, il est nécessaire de documenter les bonnes pratiques architecturales des bibliothèques pour mieux saisir les étapes, les critères et les contraintes qui régissent ces projets d'envergure. D'autre part, il faut analyser quelques cas de bibliothèques rénovées pour en tirer des leçons. Finalement, une comparaison des statistiques disponibles doit être faite afin de mieux comprendre la portée des rénovations sur les usagers des bibliothèques.

\section{Méthodologie de recherche}

Dans le cadre cette analyse, une méthodologie qualitative permettant une approche interprétative des ressources documentaires et des données statistiques a été utilisée.

Les rapports sur les bibliothèques publiques québécoises, obtenus par l'entremise du Portail de données ouvertes de la Ville de Montréal (2019) et de l'Enquête annuelle sur les bibliothèques publiques du Québec (EABP) (Bibliothèque et Archives nationales du Québec [BAnQ], s. d.), ont permis d'analyser des statistiques sur les collections, les usagers et les installations. Puisqu'aucune statistique comparative sur l'avant et l'après des bibliothèques publiques québécoises rénovées n'était disponible, les données annuelles des bibliothèques ont été interprétées de façon qualitative. Cette même approche a été adoptée à la lecture de rapports, d'articles de revue et de livres qui contenaient principalement des renseignements - parfois étayés par des données sur les bonnes pratiques architecturales en bibliothèque et sur le parcours de bibliothèques rénovées ou construites.

\section{Définition des concepts}

En vue d'éviter les ambiguïtés terminologiques, les concepts étudiés sont définis. Par rénovation, on entend une «opération visant à rafraîchir ou à moderniser un bâtiment ou ses composantes architecturales» (Ville de Lévis, 2018). Par réaménagement, terme sous-jacent à rénovation (voir figure 1), on entend l'action "[ $\left.\mathrm{d}^{\prime}\right]$ aménager d'une autre façon, [d']apporter des modifications à [un bâtiment] » (Centre national de ressources textuelles et lexicales [CNRTL], 2012). Un agrandissement implique "[l'] extension, [l'] accroissement des dimensions " (CNRTL, 2012) d'un bâtiment existant. Le terme construction (voir figure 2) sera utilisé à titre de "processus de création" (Gorse, Johnston et Pritchard, 2012) d'un nouveau bâtiment.

\section{Revue de la littérature}

Les bonnes pratiques, incluant les étapes, les critères et les contraintes des projets d'architecture des bibliothèques, ont été documentées. Certaines s'appliquent en tout lieu, indépendamment des politiques en vigueur, tandis que d'autres dépendent entièrement des lieux. Le contexte géographique du Québec étant visé par cette recherche, les pratiques qui s'y appliquent ont été priorisées, à moins que des leçons puissent en être tirées. 


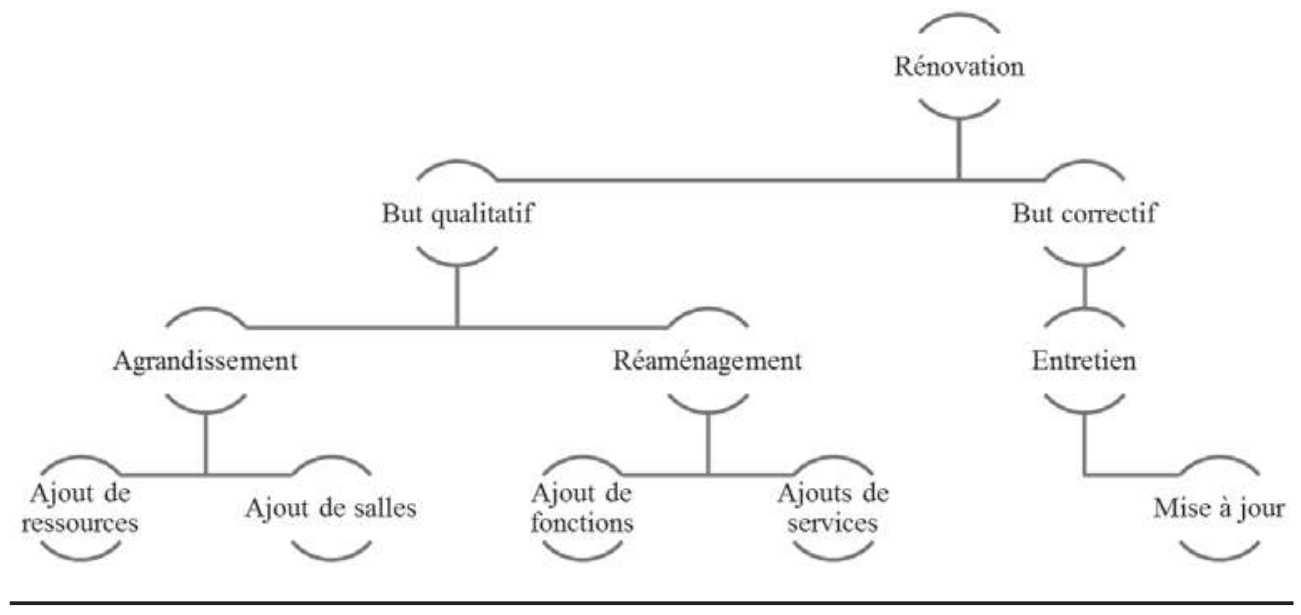

\section{FIGURE 2}

Schéma conceptuel de la construction

et de ses déclinaisons

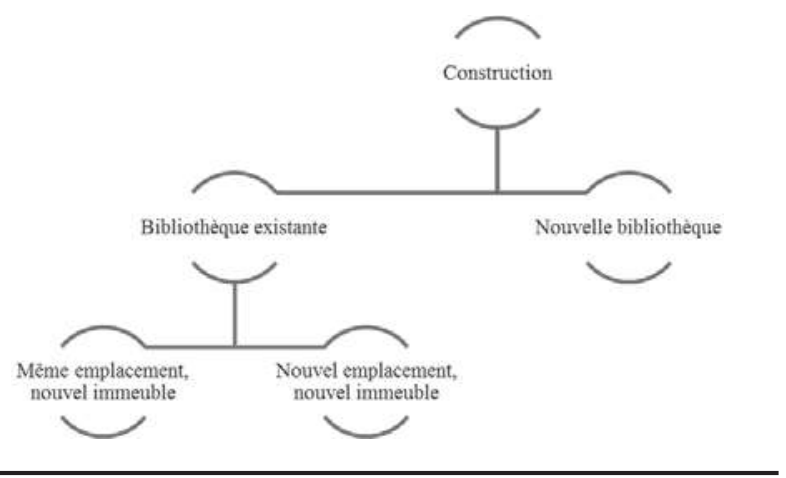

La grande majorité des ouvrages indique comme première étape la planification aussi précoce que possible (McCabe, 2000 ; Pinet, 2014) et la définition d'une vision pour le projet (Perreault, 2014; Deyrup, 2017). Puisque cette vision servira d'assise et de ligne directrice lors du concours d'architecture et tout au long des étapes de la conception, plusieurs mentionnent l'importance de sonder la population au préalable (Plante et Bissonnette, 2013; Schlipf et Moorman, 2018). Qu'il s'agisse de sondages (McCabe et Kennedy, 2003), de consultations publiques (Ordre des architectes du Québec [OAQ], 2016-2017) ou de comités de cocréation entre citoyens-usagers et bibliothécaires (Cliche et Martel, 2014), l'implication citoyenne en amont du processus veille à ce que l'espace réaménagé ou construit tienne compte des besoins des usagers (Cliche et Martel, 2014), maintenant et demain. En ce sens, «le citoyen est aussi partie prenante [...] du processus de design» (Cliche et Martel, 2014). Plante et Bissonnette soutiennent même que la «rédaction du programme architectural de la bibliothèque se fait davantage d'après l'opinion des usagers et des bibliothécaires que d'après des études de cas ou des recherches empiriques » (2013).
Ensuite vient l'élaboration du programme fonctionnel et technique (PFT) - une étape propre au Québec même si son contenu reprend certaines considérations formulées en contexte états-unien (Sannwald, 2009). Cette étape consiste à colliger les aspects fonctionnels et techniques du projet dans un document détaillé destiné aux membres du "comité exécutif» (Ville de Montréal, s. d.) ou du comité spécial chargé de mener à bien le projet (McCabe et Kennedy, 2003). Le projet de construction ou de rénovation de la bibliothèque publique sera décrit, puis mis en contexte (Ville de Montréal, 2017b). Les caractéristiques de ses aménagements intérieurs et extérieurs y seront également détaillées ainsi que d'autres exigences techniques, dont la qualité architecturale, la durabilité, les exigences environnementales et de développement durable, incluant la certification LEED (Ville de Montréal, 2017b), si elles s'appliquent au projet.

Selon Perreault, «traduire une vision aussi claire et précise que possible de chaque secteur est indispensable» (2014). En ce sens, il importe de spécifier la fonction, l'ambiance, l'éclairage, la superficie, les matériaux et l'acoustique recherchés (Perreault, 2014). La participation des bibliothécaires lors de l'élaboration du PFT et du concours d'architecture, mais également pendant toutes les étapes du processus, demeure essentielle, voire décisive (Perreault, 2014). L'implication des bibliothécaires lors du concours d'architecture leur permettra d' "apporte[r] leur expertise dans l'analyse des projets [et d']identifie[r] celui qui est le plus conforme à leur vision [pour éviter] de compromettre des fonctionnalités bibliothéconomiques" (Perreault, 2014). De surcroît, les bibliothécaires seront à même de «sensibiliser les professionnels de la ville [et les membres du jury] à la mission des bibliothèques afin qu'ils soient porteurs de celle-ci dans leurs propres équipes de travail» (Perreault, 2014). 


\section{Caractéristiques architecturales}

Les littératures québécoise et états-unienne mentionnent sept caractéristiques architecturales dont les retombées positives semblent démontrées: l'éclairage, le rayonnage, la conception de l'espace, les espaces pour l'étude ou la lecture, les espaces ouverts, l'insonorisation, les formes et la conception écoénergétique.

Le choix de l'éclairage reste primordial puisqu'il aura une incidence sur l'expérience des usagers et, potentiellement, sur la fréquentation de la bibliothèque. Comme le soulignent McCabe et Kennedy, "a successful library facility today must incorporate both the best of natural light and internal-lighting techniques to produce a truly flexible facility, one that will provide a custom visual experience for every user $^{1} »(2003$, p. 199). Schlipf et Moorman abondent dans le même sens en soutenant que la qualité de l'éclairage demeure l'une des caractéristiques les plus importantes d'une bibliothèque (2018, p. 717). D'après le Primary Research Group, les bibliothèques identifient l'amélioration de l'éclairage naturel comme critère important lors de rénovations dans 30,77\% des cas (2015, p. 50). Au Québec, la bibliothèque Raymond-Lévesque, construite à Longueuil en 2011 et lauréate du Prix d'excellence en architecture 2013 décerné par l'Ordre des architectes du Québec, a opté pour une combinaison de ces deux types d'éclairages. Depuis son ouverture, elle a observé un accroissement de la fréquentation de l'ordre de $15 \%$ (Perreault, 2014). Prétendre que cette augmentation se base principalement sur la stratégie d'éclairage serait périlleux. Cependant, puisque les espaces mal éclairés ou difficiles à éclairer ainsi que les hauts plafonds foncés sont généralement à éviter (Schlipf, 2011), il semble plausible que les bibliothèques dont l'éclairage naturel est optimisé et complété adéquatement par l'éclairage artificiel soient plus fréquentées que les autres.

Le rayonnage a un impact non négligeable sur l'accès à la collection de la bibliothèque par ses usagers (Beaudry et Lacroix, 2014). Par exemple, le choix d'un rayonnage ouvert et facilement accessible façonnera l'expérience des lecteurs, qui pourront fureter les rayons à loisir en quête de leur prochaine lecture (Beaudry et Lacroix, 2014). Ainsi, le type de rayonnage peut intégrer la sérendipité (Beaudry et Lacroix, 2014) ou la restreindre.

L'ajout d'espace pour la lecture ou l'étude lors de rénovations dans les bibliothèques publiques québécoises se manifeste avec modération (Ministère de la Culture et des Communications [MCC] et BAnQ, 2018; Ville de Montréal, 2019) en comparaison aux États-Unis, où l'ajout de places assises est considéré comme un facteur important dans 30,77\% des

1. "pour être couronnée de succès de nos jours, une bibliothèque doit marier les techniques d'éclairage naturel et artificiel de façon à créer un espace véritablement polyvalent qui offre une expérience sur mesure pour chaque utilisateur.» (Notre traduction) cas (Primary Resarch Group, 2015, p. 29-30). En contrepartie, les bibliothèques québécoises semblent privilégier l'ambiance des espaces de la bibliothèque, dont ceux destinés à la lecture. Perreault explique que l'«ambiance et la définition des différentes zones doivent contribuer à cette transformation [que procure la lecture au lecteur et que p]lus le lieu est attrayant, plus il sera fréquenté.» (Perreault, 2014)

En ce qui a trait à l'aménagement d'espaces ouverts, les bibliothèques publiques québécoises s'alignent sur la conception du troisième lieu (Cliche et Martel, 2014), qui s'inspire du four spaces - modèle scandinave qui divise l'espace en quatre parties fonctionnant en synergie: apprentissage, inspiration, rencontre, participation (Jochumsen et al., 2012). Cette intention demeure ancrée dans l'atmosphère ou l'ambiance qui vise à créer des «lieux accueillants, attrayants, fonctionnels et ouverts à la participation de la communauté» (Cliche et Martel, 2014). Le nouvel aménagement de la bibliothèque Saul-Below illustre bien le concept «[d']ouverture à une variété de types d'espace favorisant les rencontres» (Cliche et Martel, 2014), qui reprend certainement la dimension de la rencontre exprimée par le four spaces scandinave. En effet, selon le modèle scandinave, "the meeting space is an open, public space and a place between work and home where citizens can meet other people, who are both like them and differ from them ${ }^{2}$ » (Jochumsen et al., 2012, p. 592).

L'insonorisation demeure un facteur important dans 27,69\% des bibliothèques publiques états-uniennes (Primary Research Group, 2015). Qui plus est, Schlipf explique à quel point le facteur acoustique peut avoir des répercussions négatives a posteriori de la construction ou des rénovations, notamment dans le cas des plafonds cathédrale (2011, p. 242). D'après l'auteur, "many acoustic problems in libraries are due to the omission of soundabsorbing surfaces on portions of ceilings that are not flat ${ }^{3}$." (Schlipf, 2011, p. 242) C'est pourquoi il importe de déterminer «le niveau sonore souhaité» (Perreault, 2014) de chaque section de la bibliothèque dès les débuts de sa conception (Schlipf et Moorman, 2018). On évitera ainsi la transmission de bruit provenant de salles et d'escaliers, de même que la réflexion acoustique (ou les effets d'écho) liée à l'utilisation de certains matériaux, comme le verre, ou de certains types de structures comme les balcons et les atriums (Schlipf, 2011). À titre d'exemple, la bibliothèque Raymond-Lévesque a opté pour l'insertion d'une feuille de feutre derrière le panneau latéral des rayonnages comme coupe-bruit (Perreault, 2014).

\footnotetext{
2. «l'espace de rencontre est un espace ouvert et public, un lieu à mi chemin entre le travail et la maison où les citoyens peuvent rencontrer d'autres personnes qui leur ressemblent et qui sont différentes d'eux également. » (Notre traduction)

3. "plusieurs problèmes d'acoustique dans les bibliothèques sont liés à l'omission de matériau absorbant acoustique sur les sections en relief des plafonds.» (Notre traduction)
} 
À l'étape de conception du projet de rénovation ou de construction, le choix des formes pour l'espace et le mobilier est important, car celui-ci aura un impact sur d'autres éléments, dont l'insonorisation, l'éclairage et le rayonnage. Le rôle-conseil du bibliothécaire sur ce plan reste primordial, car «il [lui] arrive[ra] d'avoir à convaincre les autres [...] que certains aspects, parfois plus séduisants, risquent de compromettre des fonctionnalités bibliothéconomiques " (Perreault, 2014). D'ailleurs, Schlipf et Moorman (2018) recommandent d'éviter les formes insolites et les triangles pour privilégier les rectangles et les carrés. En effet, en dépit de leur originalité, les formes inhabituelles sont souvent difficiles à éclairer et représentent une perte d'espace, voire un danger (Schlipf, 2011). C'est notamment le cas des escaliers aux formes étranges qui ralentissent non seulement la montée et la descente, mais favorisent les chutes (Schlipf, 2011). En outre, les rayonnages non rectangulaires ne logeront pas autant de documents que les rayonnages carrés. Dans le doute, l'ingénieur du projet devrait effectuer une analyse de la valeur afin de déterminer le coût de la surface habitable par mètre carré pour éviter les mauvaises surprises (Schlipf, 2011, p. 237).

Finalement, les projets de conception durable et écoénergétique sont présents au Québec en plus d'être mentionnés dans la littérature états-unienne. Par ailleurs, au Québec, leur fréquence semble plus élevée lors de la construction de nouvelles bibliothèques que lors de la rénovation de bibliothèques existantes (Association des bibliothèques publiques du Québec [ABPQ], 2016, 2018; Portail Constructo, 2017). Même si des économies d'énergie sont à prévoir pour les bibliothèques qui optent pour les constructions écoénergétiques, il ne faut pas oublier que leur intégration entraîne un surcoût (Écohabitation, 2019; Harrison-Julien et Gerbet, 2015). Cela étant dit, à Varennes, où la nouvelle bibliothèque est certifiée LEED de niveau Or, le maire, Martin Damphousse, affirme que celle-ci utilise 78,5\% moins d'énergie que si elle avait été construite de façon conventionnelle (OAQ, 2016). Selon Edwards, «it is now widely accepted that the quality of environmental design [qui accorde une grande place à la lumière naturelle] affects the attitudes and behavior of library staff and users, particularly in areas such as productivity and concentration levels ${ }^{4}$ (2011).

\section{Études de cas}

Vingt-huit bibliothèques municipales québécoises rénovées ont été répertoriées dans le cadre de cette analyse. Douze d'entre elles ont été sélectionnées, puis regroupées en

4. «l'idée que la conception environnementale [qui accorde une grande place à la lumière naturelle] ait un impact sur l'attitude et le comportement des usagers et du personnel de la bibliothèque, notamment en favorisant la productivité et la concentration, est maintenant très répandue.» (Notre traduction) sous-groupes de populations comparables d'après le recensement 2016 de Statistique Canada (2019) et l'Annuaire statistique de la Ville de Montréal (2017a). Leurs statistiques avant, pendant et après les rénovations ont été analysées. Puisque les données publiées dans le cadre de l'Enquête annuelle sur les bibliothèques publiques (BAnQ, s. d.) ne sont pas segmentées par succursale, mais bien par municipalité, les municipalités dotées d'une seule bibliothèque publique - à l'exception de Montréal et de Repentigny - ont été retenues pour analyse. D'une part, la Ville de Montréal segmente ses statistiques par succursale, ce qui a permis l'obtention de données exactes pour les arrondissements étudiés. D'autre part, le nombre de bibliothèques rénovées de populations comparables pour le sous-groupe III (villes d'environ 84000 habitants) étant restreint, la municipalité de Repentigny a été ajoutée même si celle-ci dispose de deux bibliothèques. Brossard ne compte qu'une seule bibliothèque pour un bassin de population très semblable à Repentigny; c'est pourquoi leurs statistiques ont été comparées même si la nature de leurs rénovations diffère.

Des nombreux critères indiqués dans les statistiques de l'EABP (BAnQ, s. d.) comme du Portail de données ouvertes de la Ville de Montréal (2019), ceux qui semblaient le plus susceptibles d'afficher une différence avant et après les rénovations ont été retenus. Ainsi, le nombre de visites (ou la fréquentation), de prêts de documents en bibliothèque, d'activités et de participants à celles-ci, de places assises, la superficie de l'espace en mètres carrés et le nombre d'usagers inscrits ont été compilés et comparés, lorsque ces données étaient disponibles. Ce faisant, les statistiques de 2007 à 2017 de neuf des douze bibliothèques sélectionnées ont été analysées. Pour la période de 2011 à 2017, les données des douze bibliothèques ont pu être comparées.

De façon générale, une diminution des visites, des prêts en bibliothèque et du nombre d'usagers inscrits au moment des rénovations est constatée, suivie d'une augmentation dans la majorité des cas. Cette augmentation, tantôt modeste, tantôt significative, atteint souvent un plateau l'année suivant les rénovations ou la subséquente, ce qui pourrait s'expliquer par le retour à la normale une fois l'attrait de la nouveauté terminé.

Par exemple, dans le cas de Boucherville, on constate une diminution de $83,8 \%^{5}$ du nombre de visites l'année des rénovations (27 092 visites) par rapport à l'année précédente, suivie d'une augmentation de 982,5\% l'année suivante (293 263 visites) et d'une diminution de 39,7\% (176 922 visites) l'année subséquente. Un schéma semblable se produit au niveau du nombre de prêts en bibliothèque, qui subit une diminution de $43,1 \%$ l'année des

\footnotetext{
5. Formule utilisée pour tous les calculs : ((année sélectionnée - année précédente) / année précédente) x 100
} 
rénovations (213 690 prêts) par rapport à l'année précédente pour atteindre $81,2 \%$ d'augmentation (387 300 prêts) l'année suivante et $0,4 \%$ d'augmentation (388 750 prêts) l'année subséquente.

Le cas de Ville de Mont-Royal est similaire avec une diminution des visites de 28,6\% l'année des rénovations (115 800 visites) par rapport à l'année précédente, et 52,8\% d'augmentation (176951 visites) l'année suivante pour atteindre 5,2\% (186 239 visites) l'année subséquente. Dans l'ensemble, Brossard, Rouyn-Noranda ainsi que l'arrondissement de Lachine suivent ce schéma, si on fait omission du décalage d'un an après l'année des rénovations, qui pourrait s'expliquer par des travaux résiduels.

Pour ce qui est de Kirkland, le schéma type, quoique devancé d'une année par rapport aux villes précédentes, reste essentiellement le même avec une augmentation des visites de 38,8\% (152 860 visites) l'année des rénovations suivie de diminutions de 3,3\% l'année suivante (147837 visites) et de 4,8\% (140 674 visites) l'année subséquente. La municipalité de Candiac reprend essentiellement le même schéma avec une augmentation des visites de 3,5\% (41 164 visites) l'année des rénovations, une diminution de 9,3\% (37 320 visites) l'année suivante et une augmentation de 3,3\% (38 536 visites) l'année subséquente.

Pour ce qui est du nombre d'usagers inscrits dans les bibliothèques rénovées, huit des neuf municipalités dont les statistiques sont disponibles ont vu ce nombre augmenter l'année suivant les rénovations. À titre d'exemple, à la bibliothèque de Boucherville, le nombre d'usagers inscrits diminue de $2 \%$ l'année des rénovations (20 087 inscrits) pour augmenter de 18,4\% l'année suivante (23 781 inscrits) et diminuer de 36,6\% l'année subséquente (15 787 inscrits). Dans le cas de Ville de Mont-Royal, le nombre d'usagers inscrits passe de 4973 l'année précédant les rénovations à 6855 l'année des rénovations, ce qui représente une augmentation de $37,8 \%$.

\section{Discussion des résultats}

De façon générale, les variations de pourcentage entre les municipalités semblent liées au nombre d'habitants. Par exemple, les municipalités du sous-groupe I (environ 20000 habitants) affichent des augmentations moins spectaculaires que celles du sous-groupe II (environ 40000 habitants).

De plus, le taux de croissance annuel des populations de même que la nature des rénovations effectuées ont probablement eu une incidence sur les données recueillies par les bibliothèques analysées. Ainsi, les variations observées précédemment pourraient être attribuables à d'autres facteurs que les rénovations.
Cela dit, les tendances générales indiquent une diminution des visites et des prêts pendant les rénovations, puis une augmentation et un plafonnement l'année suivante. À quelques exceptions près, le nombre d'usagers inscrits reste plus stable, ce qui démontre l'intérêt croissant de la population pour les bibliothèques publiques. Ces tendances illustrent à quel point la visibilité des bibliothèques publiques est accrue lors de projets de rénovation. Manifestement, les citoyens apprécient les changements effectués.

Le lien entre les bonnes pratiques et les études de cas a été étudié d'après les statistiques compilées. Comme l'illustrent les bonnes pratiques architecturales (voir tableau 1), au moins cinq des douze bibliothèques rénovées se rallient à l'une des sept caractéristiques clés. En effet, le rang des bibliothèques en tête de liste est indirectement proportionnel au nombre de bonnes pratiques utilisées dans trois cas sur cinq (voir tableau 1). Autrement dit, les bibliothèques dont les projets de rénovation combinent plus d'une bonne pratique architecturale se positionnent généralement en tête de leur sous-groupe de population.

Somme toute, l'adoption de bonnes pratiques architecturales lors de rénovations semble garante de succès pour les bibliothèques. Celles-ci gagnent donc à en tirer profit pour optimiser les retombées positives sur leur communauté, notamment l'augmentation de la fréquentation et des nombres d'emprunts en bibliothèque et d'usagers inscrits. De surcroît, l'analyse des autres statistiques des cinq bibliothèques en tête de liste indique une augmentation des nombres d'activités en bibliothèque et de participants, ce qui confirme la valeur ajoutée des rénovations pour les citoyens.

Ce constat apporte dès lors des éléments de réponse aux questions à l'origine de cette analyse. Il est possible de mettre la valeur architecturale de la bibliothèque publique au service des usagers, car, au-delà de l'attrait des rénovations, la valeur ajoutée pour la population se manifeste par l'accroissement des nombres de visites, d'emprunts en bibliothèque, d'usagers inscrits, d'activités et de participants à celles-ci.

\section{Recommandations}

À la lumière des bonnes pratiques puisées dans la littérature, de leurs applications aux bibliothèques publiques du Québec et des études de cas présentées, voici quelques recommandations dont pourraient s'inspirer les bibliothèques lors d'éventuels projets de rénovation ou de construction.

D'abord, dans le cas de bibliothèques existantes, la collecte et la comparaison de statistiques avant, pendant et après les travaux de rénovation devraient faire partie intégrante du processus de conception architecturale, d'autant que ces 


\begin{tabular}{|c|c|c|c|c|c|c|}
\hline Bonnes pratiques architecturales & Type de rénovations & Ville & Gr & $\begin{array}{l}\text { Rang } \\
\text { visites }\end{array}$ & $\begin{array}{l}\text { Rang } \\
\text { prêts }\end{array}$ & $\begin{array}{l}\text { Rang } \\
\text { inscrits }\end{array}$ \\
\hline $\begin{array}{l}\text { Espaces de réunion } \\
\text { Insonorisation } \\
\text { Espace rectangulaire } \\
\text { Rayonnage rectangulaire spacieux } \\
\text { (Bibliothèque de Brossard Georgette-Lepage, } \\
2014 \mathrm{a}, 2014 \mathrm{~b} \text { ) }\end{array}$ & $\begin{array}{c}\text { Agrandissement } \\
\text { Espace SODA pour } \\
\text { adolescents (espace isolé } \\
\text { du reste de la bibliothèque) }\end{array}$ & Brossard & III & 1 & 1 & 1 \\
\hline $\begin{array}{l}\text { Conception durable } \\
\text { Espaces pour l'étude / la lecture } \\
\text { Espaces, mobilier et rayonnages rectangulaires } \\
\text { Éclairage naturel et artificiel } \\
\text { Espaces ouverts } \\
\text { (MCC et BAnQ 2018; Ville de Boucherville, 2016, 2019) }\end{array}$ & $\begin{array}{l}\text { Agrandissement } \\
\text { et réaménagement }\end{array}$ & Boucherville & $\|$ & 2 & 2 & 2 \\
\hline $\begin{array}{l}\text { Espaces la lecture } \\
\text { Espace rectangulaire } \\
\text { (Ville de Mont-Royal, 2015) }\end{array}$ & $\begin{array}{l}\text { Agrandissement } \\
\text { et réaménagement }\end{array}$ & $\begin{array}{c}\text { Ville de } \\
\text { Mont-Royal }\end{array}$ & 1 & 3 & 3 & 5 \\
\hline $\begin{array}{l}\text { Rayonnage rectangulaire spacieux qui facilite } \\
\text { la découverte } \\
\text { (Ville de Vaudreuil-Dorion, 2017) }\end{array}$ & $\begin{array}{l}\text { Agrandissement } \\
\text { et réaménagement }\end{array}$ & Vaudreuil-Dorion & $\|$ & 3 & 5 & 4 \\
\hline $\begin{array}{l}\text { Espaces pour la lecture } \\
\text { Éclairage artificiel optimisé (murs et plafonds clairs) } \\
\text { Rayonnage rectangulaire spacieux } \\
\text { Espaces ouverts } \\
\text { (Ville de Kirkland, 2018) }\end{array}$ & Réaménagement & Kirkland & I & 5 & 7 & 3 \\
\hline
\end{tabular}

données sont publiées annuellement par toutes les bibliothèques publiques du Québec. De surcroît, les statistiques compilées lors de l'Enquête annuelle sur les bibliothèques publiques devraient être segmentées par succursale et non par municipalité, dans le but de faciliter leur éventuelle analyse. En outre, les éléments compilés dans toutes les bibliothèques publiques du Québec devraient faire consensus pour éviter toute discordance d'une municipalité à l'autre, comme c'est notamment le cas entre les régions du Québec et Montréal.

D'une part, cette compilation statistique servirait de mesure quantitative aux bibliothèques a posteriori des rénovations, ce qui permettrait d'évaluer le succès du projet. D'autre part, lors de rénovations subséquentes, cette analyse statistique pourrait être annexée au programme fonctionnel et technique afin d'appuyer le nouveau projet et démontrer la valeur ajoutée potentielle pour la population. Ainsi, les bibliothèques ont intérêt à colliger et à publier les statistiques qui démontrent à quel point l'argent investi sert bien la population.

Sur le plan des caractéristiques architecturales, l'éclairage reste la plus importante d'entre elles (Schlipf et Moorman,
2018). Une place de choix lui revient donc au moment de définir la vision d'un projet. L'éclairage naturel dans les zones dédiées à la lecture et à l'étude (Edwards, 2011) - une option prisée par la conception écologique - gagnerait à être davantage intégré aux rénovations d'envergure. De plus, les aménagements qui facilitent l'ajout de services (espace ouvert ou de réunion, places assises, formes et rayonnages ergonomiques) ne sont pas à négliger, car ceux-ci attireront et garderont les usagers en bibliothèque de façon durable conjointement à l'éclairage qui, à lui seul, ne suffit pas.

\section{Limites de la recherche}

Considérant que cette analyse a été conduite d'après une approche interprétative de la littérature et des statistiques consultées, elle se voit confrontée à certaines limites sans lesquelles elle n'aurait pas pu être possible.

En premier lieu, la collection de livres et d'articles sur le sujet de l'architecture des bibliothèques reste un processus subjectif. Il est possible que certains ouvrages ou articles n'aient pas été repérés lors des recherches documentaires. L'analyse des documents lus et examinés demeure, elle 
aussi, sujette à interprétation et source de biais involontaire. Il se peut également que d'autres bibliothèques publiques rénovées ou construites au Québec depuis 2005 existent au-delà de celles répertoriées, car aucune liste exhaustive n'est apparue dans les résultats de recherche.

En deuxième lieu, les données recueillies par la Ville de Montréal (2019) et l'EABP (BAnQ, s. d.) n'étant ni uniformes ni systématiques, leur comparaison devrait être considérée à titre indicatif. Il est d'ailleurs possible que certaines des augmentations ou des diminutions commentées précédemment soient liées à d'autres facteurs tels que le nombre d'habitants, le taux de croissance annuel de la population et la nature des rénovations effectuées dans les douze bibliothèques étudiées. De plus, le type de statistiques recueillies par la Ville de Montréal diffère de celles compilées lors de l'EABP, ce qui introduit une discontinuité partielle au moment de comparer les données de ces deux sources. La sélection des douze bibliothèques publiques rénovées a été influencée par la taille des populations des municipalités afin que des milieux semblables soient comparés. À une exception près, l'absence de plusieurs bibliothèques dans la même municipalité a été privilégiée pour les bibliothèques à l'extérieur de Montréal, puisque l'EABP combine les données des bibliothèques de chaque municipalité.

\section{Conclusion}

L'analyse de douze bibliothèques publiques québécoises rénovées illustre les liens entre les bonnes pratiques architecturales et les tendances générales, notamment la diminution des nombres de visites, de prêts et d'usagers inscrits pendant les rénovations, et l'augmentation puis le plafonnement de ces nombres l'année suivante ou la subséquente. Ces données non seulement expriment l'appréciation des rénovations par les usagers, mais confirment également l'intérêt que ceux-ci portent aux bibliothèques publiques.

Bien entendu, les retombées positives de ces rénovations mériteraient une étude plus poussée afin qu'elles puissent être documentées et mesurées tant qualitativement - par le personnel des bibliothèques et par le biais de sondages que quantitativement par les municipalités, les gouvernements et l'ensemble de la société québécoise. De cette manière, l'impact positif des réaménagements et des constructions rayonnera au-delà de la sphère des bibliothèques publiques pour développer au Québec une culture de la bibliothèque qui valorise et encourage sa mise au goût du jour et au diapason de ses usagers.

\section{SOURCES CONSULTÉES}

Association des bibliothèques publiques du Québec. (2016). Rapport annuel 2014-2015. Repéré à abpq.ca/pdf/rapport_annuel_ abpq_2014-2015.pdf

Association des bibliothèques publiques du Québec. (2018). Rapport annuel 2017. Repéré à abpq.ca/pdf/rapport_annuel_abpq_2017.pdf

Beaudry, G. et Lacroix, Y. (2014). Au-delà du programme, l'architecte du bien-être: entrevue avec Manon Asselin. Documentation et bibliothèques, 60(2-3): 126-129. Repéré à erudit.org/fr/revues/ documentation/2014-v60-n2-3-documentation01444/1025509ar/

Bibliothèque de Brossard Georgette-Lepage. (2014a). Soda: espace adolescents. Repéré à bibliotheque.brossard.ca/espaces/soda

Bibliothèque de Brossard Georgette-Lepage. (2014b). Soda: espace professionnel, bibliothèque. Repéré à biblio.brossard.ca/espaces/ soda/espace-professionnel

Bibliothèque et Archives nationales du Québec [BAnQ]. (s. d.). Enquête annuelle sur les bibliothèques publiques du Québec. Repéré à http://www.banq.qc.ca/services/services_professionnels/milieux_ doc/statistiques/enquete_annuelle/index.html

Centre national de ressources textuelles et lexicales [CNRTL]. (2012). Outils et ressources pour un traitement optimisé de la langue [Ortolang]. Repéré à www.cnrtl.fr/definition/

Cliche, L. et Martel, M. D. (2014). Cinq bibliothèques de Montréal dans la société du savoir: une nouvelle génération de tiers lieux. Documentation et bibliothèques, 60(2-3): 99-104. Repéré à erudit.org/fr/ revues/documentation/2014-v60-n2-3-documentation01444/ 1025516ar/
Deyrup, M. (dir.). (2017). Creating the High-Functioning Library Space: Expert Advice From Librarians, Architects, and Designers. Santa Barbara, CA: Libraries Unlimited, an imprint of ABC-CLIO, LLC.

Écohabitation. (2019). LEED ${ }^{\circ}$ V4 pour les habitations: surcoûts de construction. Repéré à www.ecohabitation.com/leed/surcouts

Edwards, B. W. (2011). Sustainability as a Driving Force in Contemporary Library Design. Library Trends, 60(1): 190-214. doi: 10.1353/ lib.2011.0030

Gorse, C., Johnston, D. et Pritchard, M. (2012). Construction. A Dictionary of Construction, Surveying and Civil Engineering. Oxford, Angleterre: Oxford University Press. Repéré à mcgill.on.worldcat. org/oclc/841815221

Harrison-Julien, P. et Gerbet, T. (2015, 21 juin). Une bibliothèque du futur en banlieue de Montréal. Radio-Canada. Repéré à ici radio-canada.ca/nouvelle/725500/bibliotheque-futur-varennesbanlieue

Jochumsen, H., Rasmussen, C. H. et Skot-Hansen, D. (2012). The Four Spaces - A New Model for the Public Library. New Library World, 113(11): 586-597. doi : dx.doi.org/10.1108/03074801211282948

McCabe, G. B. (2000). Planning for a New Generation of Public Library Buildings. Westport, CT: Greenwood Press.

McCabe, G. B. et Kennedy, J. (2003). Planning the Modern Public Library Building. Westport, CT: Libraries Unlimited. 
Ministère de la Culture et des Communications du Québec [MCC] et Bibliothèque et Archives nationales du Québec [BAnQ]. (2018). Statistiques des bibliothèques publiques du Québec. Repéré à banq.qc.ca/statbib

Ordre des architectes du Québec [OAQ]. (2016). Bibliothèque municipale de Varennes: La totale. Esquisses, 27(2). Repéré à www.oaq. com/esquisses/mdb_bim/actualites/bibliotheque_municipale_ de_varennes.html

Ordre des Architectes du Québec [OAQ]. (2016-2017). Participation citoyenne: Maîtres chez nous. Esquisses, 27(4). Repéré à www. oaq.com/esquisses/identite_culturelle/dossier/participation_ citoyenne.html

Perreault, M. (2014). La Bibliothèque Raymond-Lévesque: Prix Architecture 2011. Documentation et bibliothèques, 60(2-3): 74-78. Repéré à erudit.org/fr/revues/documentation/2014-v60-n2-3documentation01444/1025511ar/

Pinet, G. (2014). La Bibliothèque publique Laure-Conan de La Malbaie: Prix Architecture 2013. Documentation et bibliothèques, 60(2-3): 85-87. Repéré à erudit.org/fr/revues/documentation/2014-v60n2-3-documentation01444/1025513ar/

Plante, J. et Bissonnette, L. (2013). Architectures de la connaissance au Québec. Québec, QC : Publications du Québec.

Portail Constructo. (2017). La bibliothèque Paul-Mercier certifiée LEED Argent. Repéré à portailconstructo.com/actualites/bibliotheque_ paulmercier_certifiee_leed_argent

Primary Research Group. (2015). Survey of Public Library Space Redesigns : 2015 Edition. New York, NY: Primary Research Group.

Sannwald, W. (2009). Checklist of Library Building Design Considerations (5éd.). Chicago, IL: American Library Association.

Schlipf, F. (2011). The Dark Side of Library Architecture: The Persistence of Dysfunctional Designs. Library Trends, 60(1): 227-255. doi : dx.doi.org/10.1353/lib.2011.0034

Schlipf, F. et Moorman, J. (2018). The Practical Handbook of Library Architecture: Creating Building Spaces That Work. Chicago, IL: ALA Editions.
Statistique Canada. (2019). Profil du recensement: Recensement de la population de 2016. Diffusé le 8 février 2017. Repéré à www12. statcan.gc.ca/census-recensement/2016/dp-pd/prof/index. cfm?Lang $=\mathrm{F}$

Ville de Boucherville. (2016). Plan des niveaux de la bibliothèque. Repéré à https://boucherville.ca/wp-content/uploads/2016/06/ INAUG.BIBLIO_PLAN_POUR-WEB2.pdf

Ville de Boucherville. (2019). Votre bibliothèque : la lecture au cœur de la nature! Repéré à boucherville.ca/residants/bibliotheque/ votre-bibliotheque/

Ville de Kirkland. (2018). Bibliothèque Kirland Library. Repéré à biblioweb.ville.kirkland.qc.ca/iguana/www.main.cls?surl=home

Ville de Lévis. (2018). Lexique architectural. Repéré à ville.levis.qc.ca/ developpement-planification/architecture-patrimoniale/lexiquearchitectural/

Ville de Montréal. (2017a). Annuaire statistique de l'agglomération de Montréal: Population totale en 2011 et 2016. Récupéré à ville. montreal.qc.ca/pls/portal/docs/PAGE/MTL_STATS_FR/MEDIA/ DOCUMENTS/01A_POPULATION_DENSIT\%C9_2016_3.PDF

Ville de Montréal. (2017b). Bibliothèque L'Octogone: Rénovation et agrandissement. Programme fonctionnel et technique. Repéré à designmontreal.com/sites/designmontreal.com/files/20171115_ pft_octogone_sans_annexes_b_a_j.pdf

Ville de Montréal. (2019). Portail données ouvertes: Bibliothèques de Montréal - statistiques sur le prêt, les collections et la fréquentation. Repéré à donnees.ville.montreal.qc.ca/dataset/bibliothequesmontreal-statistiques

Ville de Montréal. (s. d.). Bibliothèques Montréal: Programme RAC. Repéré à ville.montreal.qc.ca/portal/page?_pageid $=4276$, $118637577 \&$ dad=portal\&_schema=PORTAL

Ville de Mont-Royal. (2015). Bibliothèque Reginald-J.-P.-Dawson. Repéré à www.ville.mont-royal.qc.ca/fr/services-residents/ bibliotheque

Ville de Vaudreuil-Dorion. (2017). Bibliothèque: Informations et horaire. Repéré à www.ville.vaudreuil-dorion.qc.ca/fr/loisirs-etculture/bibliotheque/informations-et-horaire 